\title{
Diacronie
}

Studi di Storia Contemporanea

$N^{\circ} 28,4 \mid 2016$

La voce del silenzio

\section{Cristian Vasile, Viạ̧a intelectuală şi artistică în primul deceniu al regimului Ceauşescu 1965-1974}

Vita intellettuale e artistica nel primo decennio del regime di Ceausescu 1965-1974

\section{Francesco Zavatti}

\section{(2) OpenEdition}

Journals

\section{Edizione digitale}

URL: http://journals.openedition.org/diacronie/4569

DOI: $10.4000 /$ diacronie. 4569

ISSN: 2038-0925

Editore

Association culturelle Diacronie

Notizia bibliografica digitale

Francesco Zavatti, « Cristian Vasile, Viața intelectuală şi artistică în primul deceniu al regimului Ceauşescu 1965-1974 », Diacronie [Online], № 28, 4 | 2016, Messo online il 29 décembre 2016, consultato il 24

septembre 2020. URL : http://journals.openedition.org/diacronie/4569 ; DOI : https://doi.org/10.4000/ diacronie.4569 


\title{
Diacronie
}

N. 28 | 4|2016 La voce del silenzio: intelligence, spionaggio e conflitto nel XX secolo

\section{PANORAMICA: Romania 2015}

\author{
Francesco ZAVATTI *
}

\section{VASILE, Cristian, Viaţa intelectuală şi artistică în primul deceniu al regimului Ceauşescu 1965-1974 [Vita intellettuale e artistica nel primo decennio del regime di Ceausescu 1965-1974], Bucarest, Humanitas, 2015, 282 pp.}

Cristian Vasile, ricercatore scientifico all'Istituto di Storia "Nicolae Iorga" di Bucarest, è uno dei massimi esperti di comunismo romeno. Ingaggiato dalla Commissione Presidenziale per l'Analisi della Dittatura Comunista nel 2006 come esperto del rapporto tra Stato e Chiesa ortodossa nel periodo comunista, dal 2010 ha pubblicato una serie di opere dedicate allo studio dei meccanismi di controllo, burocratici ed economici, che il regime comunista ha imposto sul mondo della cultura sin dalla sua nascita nel 1948, tra repressione, censura e cooptazione degli intellettuali. Nel 2010 ha pubblicato Literatura şi artele în România comunistă, 1948-19531 [Letteratura e arte nella Romania comunista], nel 2011 Politice culturale comuniste în timpul regimului Gheorghiu-Dej ${ }^{2}$ [Le politiche culturali comuniste nel tempo del regime di Gheorghiu-Dej]. Il presente volume è l'ultimo della trilogia ed è incentrato sui primi nove anni del regime guidato da Nicolae Ceauşescu, cioè da un punto di continuità (1965) ad un punto di rottura (1974). Nel 1965, Ceauşescu ereditò un sistema di politiche culturali che Gheorghiu-Dej e la sua cerchia avevano ricevuto dal regime sovietico, messo a punto ed adattato alle esigenze locali e alla politica internazionale del partito. Sin dalla metà degli anni Sessanta, questo sistema venne potenziato coinvolgendo attivamente la popolazione: l'esempio più conosciuto, il festival popolare

1 VASILE, Cristian, Literatura şi artele în România comunistă, 1948-1953, Bucarest, Humanitas, 2010.

${ }^{2}$ VASILE, Cristian, Politice culturale comuniste în timpul regimului Gheorghiu-Dej, Bucarest, Humanitas, 2011. 
Cântarea României, organizzato dal regime, è da Vasile contestualizzato in seno al rapporto tra regime ed arti - ed a ragione: il culto di Ceauşescu crebbe a dismisura negli anni Settanta, grazie a migliaia di canzoni scritte in onore del Conducător da dilettanti che partecipavano a un concorso di musica che, apparentemente, poco o nulla aveva a che fare con la politica.

A differenza dei due precedenti volumi della trilogia, Vasile compie un'incursione nel campo della public history tentata dal regime con massimo sforzo economico ed organizzativo. La storia del Museo di Storia della Romania Socialista ben esemplifica i continui cambi di rotta del regime: mentre negli anni Sessanta Ceauşescu potenziò la strategia di cooptazione degli storici professionisti inaugurata da Gheorghiu-Dej, verso la fine degli anni Sessanta il segretario generale del partito aveva deciso di non ascoltare i pareri degli esperti per questioni propriamente tecniche, arrivando anche a ignorare gli allarmanti pareri concernenti la stabilità dell'edificio del Museo.

In conclusione, il libro di Vasile è un ottimo volume per la contestualizzazione e la problematizzazione della vita artistica ed intellettuale di un regime ancora troppo vicino nel tempo per poter essere compreso, forse, pienamente. Ma l'apertura degli archivi del partito, di cui Vasile è frequentatore assiduo, ha permesso all'autore di addentrarsi in un passato prossimo ancora in gran parte inaccessibile.

CLARK, Roland, Sfântă tinereţe legionară. Activismul fascist în România interbellica [Sacra gioventù legionaria. Attivismo fascista nella Romania tra le due guerre], Iaşi, Polirom, 2015, 288 pp.

La monografia di Roland Clark dedicata allo studio del Movimento Legionario è un volume del quale si è sentita la necessità: fino ad oggi la storiografia (internazionale e romena) sull'antropologia politica di un movimento dichiaratamente antisemita e anticomunista che combinava elementi militari alla mistica cristiano-ortodossa ha saputo solamente tracciare una narrativa del tatticismo politico della legione. Il libro di Clark si muove su un vasto, sterminato, elenco di fonti primarie: documenti provenienti da vari archivi, periodici, libri ed articoli scritti da appartenenti al movimento, memorie, diari e testimonianze orali.

La trattazione della storia del movimento legionario, nelle pagine di Clark, avviene "dal basso", chiedendosi innanzitutto cosa spinse migliaia di giovani romeni ad abbracciare una fede politica. La risposta a questo quesito è data dall'attività pratica: la praxis viene in questo volume privilegiata rispetto all'astrazione ideologico/propagandistica del movimento (e ai proclami degli intellettuali che hanno 
dichiarato simpatia per il legionarismo). La prassi, secondo Clark, ha contribuito a definire la specifica soggettività fascista del movimento: l'attivismo ha contribuito a definire la politica del movimento e le relazioni dei suoi membri, radicalizzandole progressivamente nella lotta contro lo Stato, contro i comunisti e contro gli ebrei. Sempre la prassi ha dimostrato che, mentre la Chiesa ortodossa prendeva ufficialmente le distanze dal movimento, i preti non negavano la propria benedizione alle liturgie pubbliche legionarie. Un'interessante conclusione riguarda la simpatia riscossa del movimento tra la popolazione romena: più che la violenza esercitata, elemento chiave dell'azione politica, è stata la violenza subita dal movimento da parte dello Stato romeno a innescare la simpatia della popolazione. Il fascismo, più che un repertorio di ciarpame ideologico, va - secondo Clark - compreso per quello che è: un insieme di pratiche che costruisce un'identità sociale.

\section{VELIMIROVICI, Felician, Istorie şi istorici în România comunistă, 1948-1989 [Storia e storici nella Romania comunista], Cluj-Napoca, Editura Mega, 2015, 330 pp.}

Felician Velimirovici, giovane dottore di ricerca, ha presentato un volume ricco ed esaustivo su un tema che ha appassionato gli storici romeni fin dal 1989: il rapporto tra regime comunista e disciplina storica. Velimirovici descrive rapidamente la metodologia adottata e le fonti per poi proporre al lettore un'opera che descrive minuziosamente le peripezie della storiografia romena durante l'epoca stalinista (19481955), i tentativi di cooptazione, controllo ed integrazione del "fronte degli storici" (1955-1964), la normalità contraffatta del primo decennio dell'era Ceauşescu (19651974) e un tentativo di inquadrare storicamente la storiografia romena degli anni Ottanta. Inoltre, il volume include due casi di studio originali ed interessanti: lo studio del Congresso Internazionale di Scienze Storiche a Bucarest, nel 1980, e una biografia dello storico e dissidente Vald Georgescu, "storico esemplare”.

Chiara ed efficace è l'esposizione di un'opera ricca, quasi enciclopedica. Molti sono i materiali d'archivio originali che portano l'attenzione su problemi irrisolti - ad esempio, per gli anni Ottanta, il progetto di un gigantesco istituto centralizzato per la disciplina storica che avrebbe dovuto sostituire tutti gli altri istituti storici: progetto misteriosamente voluto dal leader ma poi, sempre su richiesta del leader, altrettanto misteriosamente affossato. Nel complesso, è un'opera degna di fare parte del filone inaugurato da Vlad Georgescu. 
BOIA, Lucian, Omul şi clima. Teorii, scenarii, psihoze [L'uomo e il clima. Le teorie, gli scenari, la psicosi], Bucarest, Humanitas, 2015, e-book

La storia dell'immaginario non conosce confini temporali né fisici: li crea, contestualizzando e decostruendo le mitologie. Lucian Boia, tra i pionieri della storia dell'immaginazione umana e maestro nella sua decostruzione, si chiede: quali sono stati, nei secoli e ancora oggi, i meccanismi psicologici ed ideologici che hanno fatto sì che il genere umano fosse influenzato dal clima meteorologico e dagli eventi naturali in generale?

La neve, il freddo, il caldo, le inondazioni, i terremoti, le valanghe e la siccità sono stati (e sono ancora) oggetto di riflessione sulla condizione umana da parte dei filosofi, come ostacoli non-politici della creazione di comunità, Stati ed imperi. Il determinismo dei filosofi del Diciottesimo secolo (Montesquieu, Hume ed Herder) lasciò spazio nel Ventesimo secolo al possibilismo: storici e geografi ebbero agli inizi del Novecento un ruolo fondamentale in questo nuovo orientamento. In particolare, Ellsworth Huntington fu il primo geografo a scrivere (nel 1907) di cambiamenti climatici e delle sue conseguenze per le civilizzazioni. Oggi, più che allora, il cambiamento climatico è oggetto di discussioni politiche ai massimi livelli. Il passato della storia del clima è complicato, ma ancora più complicato è il nostro futuro, incerto tra un'idea di progresso continuo dove natura e clima vengono domati dal genere umano, e un ritorno allo stato di natura causato una grande, immaginifica catastrofe.

\section{PÂRVULESCU, Ioana (ed.), Şi eu am trăit în comunism [E ho vissuto sotto il comunismo], Bucarest, Humanitas, 2015, 408 pp.}

Ivan Denisovic è esistito anche in Romania. Il volume, costituito da racconti memorialistici sull'epoca comunista, è un necessario contributo per contestualizzare una storia altrimenti paralizzante e normativa: «sono venuti e verranno», scrive la curatrice del volume «storici, sociologi, specialisti di tutti i tipi. Conteranno i morti e i vivi, compareranno i costi immediati e a lungo termine, analizzeranno le cause e gli effetti e faranno uno schema sintetico della Storia. Schema dal quale mancherà un singolo elemento: noi, “uomini dell'Est”, più esattamente della Romania»3[1]. Questo punto di vista è importante: la memoria contribuisce non solo a dare un senso al passato, ma anche a completare e contestualizzare, con elementi che per la "Grande

\footnotetext{
3 PÂRVULESCU, Ioana (ed.), Şi eu am trăit în comunism, Bucarest, Humanitas, 2015, p. 9.
} 
Storia" sono irrilevanti, qualunque narrazione storica - altrimenti disumanizzante e, in pratica, inutile e ridicola. Il "banale" raccontato in questo volume è, invece, da prendere sul serio: offre una serie di spettacoli quotidiani involontari, a volte grotteschi e a volte drammatici, necessari per comprendere che tipo di vita hanno vissuto i romeni per quarant'anni.

\section{VINTILĂ-GHIŢULESCU, Constanța, Patimă şi desfătare. Despre} lucrurile mărunte ale vieții cotidiene în societatea românească 1750 1860 [La passione e la gioia. Sulle piccole cose della vita quotidiana nella società rumena 1750-186o], Bucarest, Humanitas, 2015, 488 pp.

L'autrice propone in quest'opera originale ed innovativa uno studio dell'universo quotidiano della Valacchia e Moldavia a cavallo tra il Diciottesimo ed il Diciannovesimo secolo. Il libro si basa su una vasta gamma di fonti archivistiche ed è diviso in sette capitoli, dedicati a svariati temi che forniscono uno spaccato della società romena del tempo: dalle norme di comportamento nella società del tempo, alle pratiche di igiene personale e ai canoni estetici in uso, dalle usanze culinarie alla percezione della vita quotidiana. È, in pratica, una storia delle differenze che intercorrevano tra i ricchi ed $\mathrm{i}$ poveri: differenze sociali, economiche, politiche, percettive. Nella vita pubblica come in quella privata, il nobile era diverso dal contadino e dal soldato; le abitudini alimentari ed il senso estetico di queste categorie sociali erano universi paralleli tra loro molto distanti. Verrebbe da chiedersi, quanto di tutto questo sia cambiato nel Diciannovesimo e Ventesimo secolo con l'introduzione nella scena pubblica del progetto nazionale non molto, sembra. Un'opera che meriterebbe senz'altro di essere tradotta in italiano (ed in inglese). Per chi non conosce il romeno, la visione del film Aferim! ${ }^{4}$ di cui l'autrice è stata consulente storica, e che tratta dello stesso periodo storico, è senz'altro raccomandata.

\section{PETRESCU, Dragoş, Entangled Revolutions. The Breakdown of the} Communist Regimes in East-Central Europe, Bucarest, Editura Enciclopedică, 2014, 438 pp.

Perché i regimi comunisti dell'Est europeo sono caduti tutti nel 1989? Perché in quell'ordine e perché le rivoluzioni furono pacifiche, con l'eccezione della Romania?

\footnotetext{
4 RADU, Jude, Ada Solomon, Romania-Bulgaria-Repubblica Ceca-Francia, 2015, 108'. 
Dragos Petrescu spiega: fattori strutturali (la stagnante economia, la decadenza ideologica), fattori congiunturali (il Vaticano, Reagan e Gorbachev) e culture politiche nazionali sono state le principali cause della fine dei regimi comunisti. Dopo il lungo e dettagliato capitolo metodologico, Petrescu propone la sua analisi sulle rivoluzioni in Polonia, Ungheria, Repubblica Democratica Tedesca, Cecoslovacchia, Bulgaria, Romania. Secondo Petrescu, ci sono state tre configurazioni che hanno legato il monolitismo comunista all'opposizione della società e che sono state il principale motivo delle rivoluzioni: in Polonia ed Ungheria, il frazionismo delle élite politiche ha causato "rivoluzioni negoziate"; in Cecoslovacchia e nella Repubblica Democratica Tedesca il monolitismo del potere ha causato rivoluzioni non negoziate, ma pacifiche, e un colpo di stato supportato da mobilitazione popolare (in Bulgaria); il terzo caso, che si limita alla Romania, è quello di un monolitismo partitico e repressivo al quale un'opposizione non strutturata ha deciso di ribellarsi. Il volume di Petrescu è un ottimo contributo volto a comprendere un momento chiave per la storia recente della regione (e per la sua comprensione a livello internazionale, visto che la casa editrice romena ha deciso di pubblicarlo in lingua inglese). 


\section{* L'autore}

Francesco Zavatti (1982), ha conseguito il dottorato di ricerca in Storia presso il Centre for Baltic and East European Studies della Södertörn University, Svezia. Si è laureato in storia (magna cum laude) presso l'Università degli Studi di Modena e Reggio Emilia con una tesi dal titolo Storiografia e Nazionalismo nella Romania di Ceauşescu. Nel periodo 2008-2011, ha collaborato a progetti educativi ed editoriali con l'Istituto della Resistenza e della Storia Contemporanea di Modena. È stato insignito nel 2010 di una borsa di studio da parte della Fondazione Cassa di Risparmio di Modena per condurre un progetto di carattere storico sull'Associazione Nazionale fra Mutilati e Invalidi di Guerra, culminati con la pubblicazione di una monografia Mutilati ed invalidi di guerra: una storia politica. Il caso modenese (Milano, Unicopli, 2011) e una mostra itinerante. Nel 2012, ha ricevuto una borsa di studio dalla Helge Axelsson Johnsons Stiftelsen (Svezia) per condurre ricerche archivistiche nell'Europa dell'Est, nell'ambito del suo dottorato. Ha pubblicato la sua seconda monografia, Comunisti per caso (Reggio Emilia, Mimesis, 2014).

URL: < http://www.studistorici.com/2014/o1/o6/francesco-zavatti/ >

\section{Per citare questo articolo:}

ZAVATTI, Francesco, «Panoramica: Romania 2015», Diacronie. Studi di Storia Contemporanea : La voce del silenzio: intelligence, spionaggio e conflitto nel XX secolo, 29/12/2016,

URL:< http://www.studistorici.com/2016/12/29/romania_numero_28/ >

Diacronie Studi di Storia Contemporanea 3 www.diacronie.it

Risorsa digitale indipendente a carattere storiografico. Uscita trimestrale. redazione.diacronie@hotmail.it

Comitato di redazione: Jacopo Bassi - Luca Bufarale - Antonio César Moreno Cantano - Deborah Paci - Fausto Pietrancosta - Alessandro Salvador - Matteo Tomasoni - Luca Zuccolo

Diritti: gli articoli di Diacronie. Studi di Storia Contemporanea sono pubblicati sotto licenza Creative Commons 3.0. Possono essere riprodotti e modificati a patto di indicare eventuali modifiche dei contenuti, di riconoscere la paternità dell'opera e di condividerla allo stesso modo. La citazione di estratti è comunque sempre autorizzata, nei limiti previsti dalla legge. 